\title{
Mechanical Properties of Boehmite Evaluated by Atomic Force Microscopy Experiments and Molecular Dynamic Finite Element Simulations
}

\author{
J. Fankhänel, ${ }^{1}$ D. Silbernagl, ${ }^{2}$ M. Ghasem Zadeh Khorasani, ${ }^{2}$ B. Daum, ${ }^{1}$ A. Kempe, ${ }^{1}$ \\ H. Sturm, ${ }^{2}$ and R. Rolfes ${ }^{1}$ \\ ${ }^{1}$ Institute of Structural Analysis, Leibniz Universität Hannover, Appelstraße 9A, 30167 Hannover, Germany \\ ${ }^{2}$ Bundesanstalt für Materialforschung und -prüfung, Unter den Eichen 87, 12205 Berlin, Germany \\ Correspondence should be addressed to J. Fankhänel; j.fankhaenel@isd.uni-hannover.de
}

Received 14 July 2016; Revised 6 October 2016; Accepted 16 October 2016

Academic Editor: Victor M. Castaño

Copyright (c) 2016 J. Fankhänel et al. This is an open access article distributed under the Creative Commons Attribution License, which permits unrestricted use, distribution, and reproduction in any medium, provided the original work is properly cited.

Boehmite nanoparticles show great potential in improving mechanical properties of fiber reinforced polymers. In order to predict the properties of nanocomposites, knowledge about the material parameters of the constituent phases, including the boehmite particles, is crucial. In this study, the mechanical behavior of boehmite is investigated using Atomic Force Microscopy (AFM) experiments and Molecular Dynamic Finite Element Method (MDFEM) simulations. Young's modulus of the perfect crystalline boehmite nanoparticles is derived from numerical AFM simulations. Results of AFM experiments on boehmite nanoparticles deviate significantly. Possible causes are identified by experiments on complementary types of boehmite, that is, geological and hydrothermally synthesized samples, and further simulations of imperfect crystals and combined boehmite/epoxy models. Under certain circumstances, the mechanical behavior of boehmite was found to be dominated by inelastic effects that are discussed in detail in the present work. The studies are substantiated with accompanying X-ray diffraction and Raman experiments.

\section{Introduction}

Aluminum oxides exhibit a wide range of commercial applications and are commonly used as a material for the reinforcement phase of nanocomposites (e.g., Chen et al. [1], Horch et al. [2], and Pradhan et al. [3]). Also, aluminum oxyhydroxides, like boehmite, show promising results as nanofillers (e.g., Arlt [4], Chen et al. [5], and Özdilek et al. [6]). In particular, Arlt [4] showed that unmodified and taurine modified boehmite nanoparticles are able to improve matrix-dominated properties of carbon fiber/epoxy composites, like shear strength, shear modulus, compressive strength, or compression after impact resistance by 10 to $25 \%$. In contrast, Shahid et al. [7] investigated boehmite particles with different surface modifications (lysine and para-hydroxybenzoate) in a carbon fiber/epoxy composite and reported an increase of tensile and flexural properties for very small particle fractions $(<2 \mathrm{wt} . \%)$. For fractions greater than approximately $5 \mathrm{wt} . \%$, the named properties decrease compared to the unfilled composite.

Comparing the results of the aforementioned studies, it is apparent that the composition of matrix, particle, and particle surface modification is crucial for the resulting composite properties. The long term goal of the presented investigations is to optimize the mechanical properties of nanocomposites and to understand the underlying mechanisms of the particle reinforcement by means of numerical methods (virtual material design). Therefore, knowledge about material properties of boehmite, namely, Young's modulus, Poisson's ratio, and density, are needed in order to model the material as realistically as possible and to calibrate the simulation models.

The structural properties of boehmite have been widely studied: experimentally through X-ray diffraction by Bokhimi et al. [8] or by means of Raman spectroscopy by Kiss et al. [9] and numerically through quantum mechanics calculations by Tunega et al. [10] and Noel et al. [11]. However, 

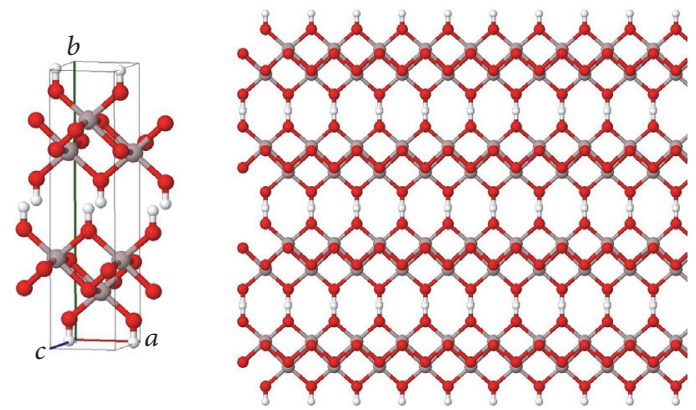

Figure 1: Crystal structure of boehmite (unit cell and material excerpt). Pictures of atomistic structures created with Jmol [14].

little literature on the mechanical properties of boehmite is available, especially concerning Young's modulus. Streller [12] reported a value of approximately $120 \mathrm{GPa}$ from nanoindentation tests using a sample of APYRAL AOH20 (Nabaltec AG) boehmite with a diameter of $1 \mu \mathrm{m}$. However, the applicability of nanoindentation of boehmite is questionable. As discussed in Section 2, monocrystalline boehmite does not appear to be stable in sufficient size to perform nanoindentation measurements. The diameter of the boehmite crystal should be at least in the higher $\mu \mathrm{m}$ range, since the maximum indentation depth should not exceed $10 \%$ of the sample height in order to get undisturbed results. Additionally, the sample surface is assumed to be an infinite plane, which allows for a region of inelastic deformation in the proximity of the indent. To the authors' best knowledge, there are no measurements of the mechanical properties of verifiable monocrystalline boehmite to be found in literature, which is apparently due to the fact that macroscopic monocrystalline boehmite is very hard to obtain.

Tunega et al. [10] calculated the bulk modulus of boehmite using density functional theory and density functional based tight binding method obtaining values of $93 \mathrm{GPa}$ and $82 \mathrm{GPa}$, respectively. Assuming, for simplicity, an isotropic behavior and a Poisson's ratio of 0.22 , Young's modulus can be estimated to fall within the range of 138 to $156 \mathrm{GPa}$.

Due to the lack of literature, the goal of the present study is the determination of the mechanical properties of boehmite. Since both approaches, experimental and numerical, have obstacles to overcome, a combination of these methods appears most promising.

\section{Boehmite}

Boehmite is a mineral of aluminum with an orthorhombic unit cell ( $a=3.693 \AA, b=12.221 \AA$, and $c=2.865 \AA)$, classified as oxyhydroxide $(\gamma-\mathrm{AlO}(\mathrm{OH}))$. Its crystal structure, shown in Figure 1, consists of double layers of oxygen octahedrons with a central aluminum atom. The outfacing oxygen is bonded via hydrogen bonds to the hydroxyl group of the adjacent layer of octahedrons [13].

Due to the weak bonds, boehmite is prone to intercalation, that is, the inclusion of small molecules, usually water, in between these layers. This causes a larger spacing in [010] direction and a perfect cleavage perpendicular to the general direction of the hydrogen bonding. Boehmite has a preferred growing direction in the $a-c$ plane, which corresponds to the (010) plane [15] and is also prone to dissolving [16, 17]. Therefore, monocrystalline boehmite is not stable in $\mathrm{mm}$ range and the boehmite crystal shows a high susceptibility to lattice defects, typically slit-like cracks and domains of amorphous boehmite. Boehmite with an increased spacing in the [010] direction is referred to as pseudoboehmite and amorphous boehmite, is usually referred to as gel. Boehmite can be found in nature or precipitated and grown from solution of aluminum salts and alumina under hydrothermal conditions.

The point of origin for the studies presented in the following was the investigation of commercially available spray dried boehmite nanoparticles (HP14 Sasol, Germany) [18] for their mechanical properties (the corresponding measurements are shown in Section 3.3). The results indicated a conspicuously lower stiffness than expected. Three possible explanations for the low stiffness are considered and discussed in the present work:

\section{(1) Effects related to the crystal size \\ (2) Slippage of weakly linked boehmite sheets \\ (3) Presence of amorphous boehmite domains.}

In order to distinguish between these effects, two additional boehmite samples of different morphology were investigated. These complementary samples feature substantial differences in terms of crystallinity and sample size, thus, providing evidence of the nature of each effect on the stiffness of the particle samples. The first complementary sample is a geological boehmite sample that comprises crystalline domains in a $\mu \mathrm{m}$ range. In this case, effects related to the sample size and influence of an amorphous phase can be ruled out, leaving the slippage of weakly linked sheets. The second complementary sample, a hydrothermally formed boehmite film, which consists predominately of pseudoboehmite and amorphous boehmite, was examined in order to investigate the effect of presence of those phases.

2.1. Spray Dried Nanoparticles HP14. Commercially available spray dried boehmite nanoparticles (HP14 Sasol, Germany) were prepared in a polymer matrix (Epoxy Araldite ${ }^{\circledR}$ LY 3585). In order to determine the morphology of the nanostructured boehmite, XRD and Raman spectroscopy were used. Results are shown in Section 3.1 and discussed later. Mechanical measurements are shown in Section 3.3.

2.2. Geological Boehmite. Boehmite crystals were prepared from a geological sample (denoted as GeoB, on Nathrolith, Saga Quarry, Tveidalen, Norway) and mounted on a glass substrate. The crystal is roughly $80 \mu \mathrm{m}$ in diameter. Since the plane of the preferred crystal growth direction is the largest flat area of the crystal, the double octahedral sheets are parallel to the substrate surface and, therefore, the measurement is taken approximately in [010] direction, that is, perpendicular to the (010) plane. The boehmite structure was confirmed by Raman spectroscopy, as shown in Section 3.2. Mechanical measurements are shown in Section 3.3. 
2.3. Hydrothermally Synthesized Boehmite. Additional boehmite samples were made via a hydrothermal route (denoted as hyB). Boehmite was synthesized on aluminum disks (diameter $20 \mathrm{~mm}$, thickness $2 \mathrm{~mm}$ ), which were cleaned (acetone in ultrasonic bath for $10 \mathrm{~min}$ ), rinsed, and polished. Okada et al. [17] established a correlation between temperature and $\mathrm{pH}$ value of a hydrothermal process and the formation of either bayerite, boehmite, or AlOOH gel. Based on these findings, the discs were exposed to purified water at approximately $120^{\circ} \mathrm{C}$ (approx. $2000 \mathrm{mbar}$ ) for at least one hour, in order to synthesize boehmite with a low degree of crystallinity. It was found that after one hour boehmite could be detected by Raman spectroscopy as shown in Section 3.2. Mechanical measurements are shown in Section 3.3.

\section{Experimental Part}

3.1. XRD Measurements. In order to determine the size of the boehmite crystals and also the crystallinity of HP14 nanoparticles, X-ray diffraction (XRD) measurements were conducted. Results are shown in Figure 2. XRD patterns were collected with $\mathrm{Cu} \mathrm{K} \alpha(\lambda=1.54056 \mathrm{~nm})$ radiation on a D8 Discover diffractometer (Bruker AXS, Germany) equipped with a Lynxeye detector. Samples were measured in transmission geometry in a $2 \theta$ range from $5^{\circ}$ to $80^{\circ}$ with a step size of $0.009^{\circ}$. For structure solutions, the measurement time per step was $1 \mathrm{~s}$.

The crystal size of the nanoparticles was determined by the Scherrer Formula [19] to be approximately $13 \mathrm{~nm}$. Also in Figure 2, the boehmite standard measurement available in the Rruff database (ID\# R120123) is shown [20]. As can be seen in the inset of Figure 2, there is a shift of the (020) reflection peak to lower values of $2 \theta$, which is due to a larger spacing between the octahedral double planes. This larger spacing is the main characteristic of pseudoboehmite [16]. The XRD measurement shows intensity besides the actual peaks. This is due to a halo of an amorphous phase which is, therefore, also present in the nanoparticles.

3.2. Raman Spectroscopy. Raman measurements of all boehmite samples were performed on a custom-made WITec Raman microscope utilizing $543 \mathrm{~nm}$ Nd:YAG-based laser and Peltier-cooled CCD spectrometer (ANDOR, DV401BV-352). A connecting fiber with $100 \mu \mathrm{m}$ core and 20x objective were used for the presented measurements. All Raman spectra were performed as qualitative measurements, meaning that only the Raman shift was evaluated, in order to identify chemical species and to determine morphologies of boehmite. The Raman intensities are not normalized. The main vibrational transitions were reported by Kiss et al. [9]. The most intense peak at $369 \mathrm{~cm}^{-1}$ is the dominant spectral signature of boehmite. In the presented measurements, this spectral signature was found at $368 \mathrm{~cm}^{-1}$, which is in satisfactory agreement with the literature value. Doss and Zallen [16] described this characteristic vibrational transition as a fully symmetric mode, in which all aluminum and oxygen atoms move parallel to the [010] direction. This movement has an intralayer-shear bond-bending character. A decrease in intralayer bond strength due to the presence of

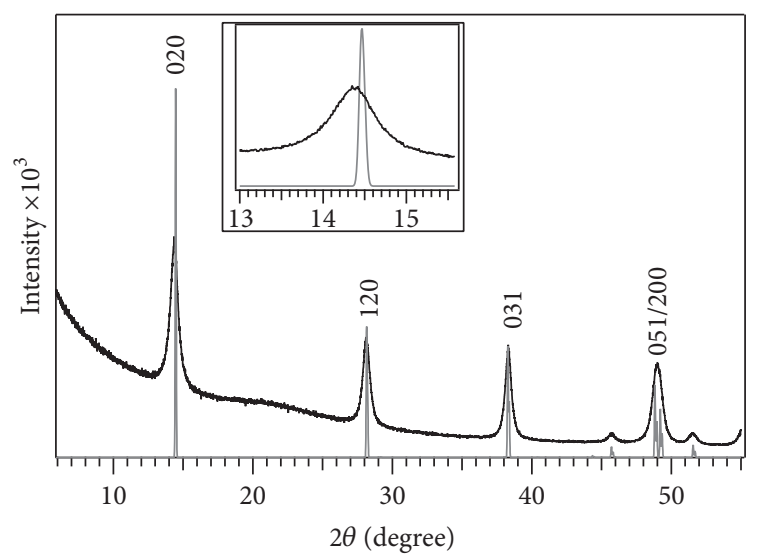

FIgURE 2: XRD measurement of HP14 (black). Also the Rruff standard spectrum (grey) is shown.

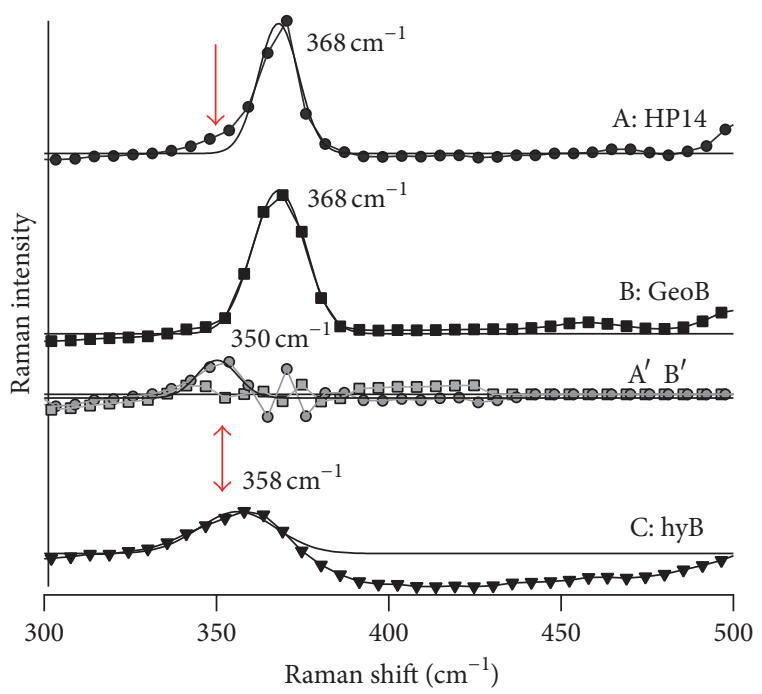

FIGURE 3: Raman intensity at $368 \mathrm{~cm}^{-1}$ Raman shift (filled markers) with their Gaussian fit (black line): $\mathrm{A}^{\prime}$ and $\mathrm{B}^{\prime}$ show the residue of the fit (grey markers).

pseudoboehmite, amorphous phases, and nanoscopic crystal sizes causes a downshift of this characteristic peak.

Considering the Raman spectrum of the hyB (C, triangle in Figure 3), the dominant peak at $368 \mathrm{~cm}^{-1}$ is significantly shifted to lower wavenumbers $\left(358 \mathrm{~cm}^{-1}\right)$. Doss and Zallen [16] found a strong relationship between the crystalline size of boehmite and such a shift, which was confirmed by Yang and Frost [21]. Since this peak is associated with lattice movements, the lack of long range order in the crystal can lead to a shift in the vibrational mode. Therefore, it can be assumed that the synthesized boehmite's morphology has indeed a very low degree of crystallinity.

Figure 3 shows also the Raman spectra of HP14 (A, filled circles) and GeoB (B, filled squares). A Gaussian fit of these peaks yields a Raman shift of $368 \mathrm{~cm}^{-1}$ in both cases. The residue of the Gaussian fit of the HP14 spectrum ( $\mathrm{A}^{\prime}$ grey circles) shows a clear shoulder peak of low intensity 
TABLE 1: AFM probe properties and summary of experimental results.

\begin{tabular}{lccccc}
\hline Sample & $k_{c}(\mathrm{~N} / \mathrm{m})$ & $\omega_{0}(\mathrm{kHz})$ & $R(\mathrm{~nm})$ & $E_{\text {boehmite }}(\mathrm{GPa})$ & Inelastic deformations \\
\hline HP14 & 14 & 202 & 17 & $4<E<70$ & Unknown \\
GeoB & 50 & 320 & 40 & $4<E<70$ & No \\
hyB & 16 & 212 & 19 & $E<4$ & Yes \\
\hline
\end{tabular}

at $350 \mathrm{~cm}^{-1}$. Such a peak is a strong indication for the presence of pseudoboehmite or amorphous boehmite in the nanoparticles. The Raman spectrum of GeoB shows a symmetrical peak at $368 \mathrm{~cm}^{-1}$, which means that there is no significant residue of the Gaussian fit of the GeoB spectrum ( $\mathrm{B}^{\prime}$ grey squares). Therefore, the lattice of GeoB can be assumed to be free of any substantial amount of defects.

3.3. AFM Measurements. All Atomic Force Microscopy (AFM) measurements were carried out with an MFP-3D microscope (Asylum Research, Santa Barbara, CA). Three different AFM modes were used: tapping mode (topography), force-distance curves (FDC), and intermodulation AFM (ImAFM) with the resulting amplitude-dependent force spectroscopy (ADFS) for mechanical measurements. For ADFS measurements, an additional intermodulation lockin analyzer by Intermodulation Products (Segersta, Sweden) was used as part of the AFM setup.

In this study, FDCs were chosen as a well-established method $[22,23]$ to determine the mechanical properties of boehmite. FDCs have been shown to be a reliable tool to obtain mechanical properties, by probing very little sample volume. This is important, since monocrystalline boehmite can only be obtained in small quantities, too little for other methods, for example, nanoindentation.

In order to record a FDC, a microfabricated tip with a radius $R$, which is mounted on a cantilever with a spring constant $k_{c}$, is used as a probe. Since the cantilever is assumed to behave linearly and elastically, Hook's law relates the applied force $F$ to the cantilever deflection $\delta: F=k_{c} \times \delta$.

The sample deformation $D$ can be calculated from the deflection $\delta$ and the position of the sample $Z$ in $Z$-direction, which is controlled by the AFM's $Z$-piezo: $D=Z-\delta$.

From a fit of the Hertz equation (1), using the relation for the reduced Young's modulus $E_{\text {tot }}(2)$ with Poisson's ratio $v$, the tip radius $R$ can be determined, when Young's modulus $E$ is known, and vice versa [24].

$$
\begin{aligned}
D & =\left(\frac{F}{\sqrt{R} \times E_{\text {tot }}}\right)^{2 / 3}, \\
\frac{1}{E_{\text {tot }}} & =\frac{3}{4}\left(\frac{1-v_{\text {tip }}^{2}}{E_{\text {tip }}}+\frac{1-v^{2}}{E}\right) .
\end{aligned}
$$

Reference measurements on glass substrates were used for this purpose since the mechanical properties of glass are well known $(E=70 \mathrm{GPa}, v=0.3)$. Once the tip radius $R$ is known, the experimental parameters are set and Young's modulus of any sample can be estimated.
In order to probe even smaller sample volumes, dynamic AFM methods can be implemented. The advantage of dynamic methods, for example, tapping mode, is that they yield mechanical information by applying very little force. One further method is the ImAFM yielding amplitudedependent force spectroscopy (ADFS). By exciting the AFM cantilever with two frequencies close to the resonance frequency, the resulting oscillation of the cantilever is a beat. The spectrum of amplitudes in a beat equals a force spectrum which can be used to probe the sample, since the amplitude of the cantilever oscillation is correlated to the force by Hooke's law. One image point is recorded per beat while the sample surface is scanned with this method. The damping of the beat's amplitude at every image point gives rise to (a) the sample height comparable to a tapping mode scan and (b) an equivalent of a FDC, namely, the ADFS curve, which can be derived from a model. This model is described in detail in [25].

For AFM measurements of HP14, the nanoparticles were embedded in an epoxy matrix. The composite was cut, polished, and cleaned with oxygen plasma, in order to remove polymer residue from the particle surface. The AFM probe used for this ADFS measurement was a Mikromasch HQ:NSC35 (Wetzlar, Germany) (cf. Table 1). For comparison, additional ADFS measurements on glass substrates were conducted.

At each pixel of the image shown in the inset of Figure 4, an ADFS curve was recorded, which provides information on the mechanical properties of the sample. The particles can be distinguished from the surrounding matrix by the local morphology of the sample surface. ADFS curves corresponding to the pixels of these distinguishable regions are summed up in order to calculate an average curve. The average curves corresponding to the particle (filled circles), the epoxy matrix (blank circles), and the reference curve on glass (grey curve) are shown in Figure 4.

Young's modulus for glass is taken as $70 \mathrm{GPa}$ as described before and the one for the epoxy matrix is known from macroscopic mechanical measurements and FDCs to be approximately $3.5 \mathrm{GPa}$.

Since the slope of ADFS curves is inversely proportional to the stiffness, the following can be understood from the plot in Figure 4: the stiffness of HP14 is higher than the stiffness of the matrix. However, boehmite unexpectedly appears to be more compliant than glass:

$$
\text { 3.5 GPa }<E_{\text {boehmite }}<70 \mathrm{GPa},
$$

which is in stark disagreement with values from the literature and numerical results which are discussed further down in the text and predict $136 \mathrm{GPa} \leq E_{\text {boehmite }} \leq 267 \mathrm{GPa}$. 


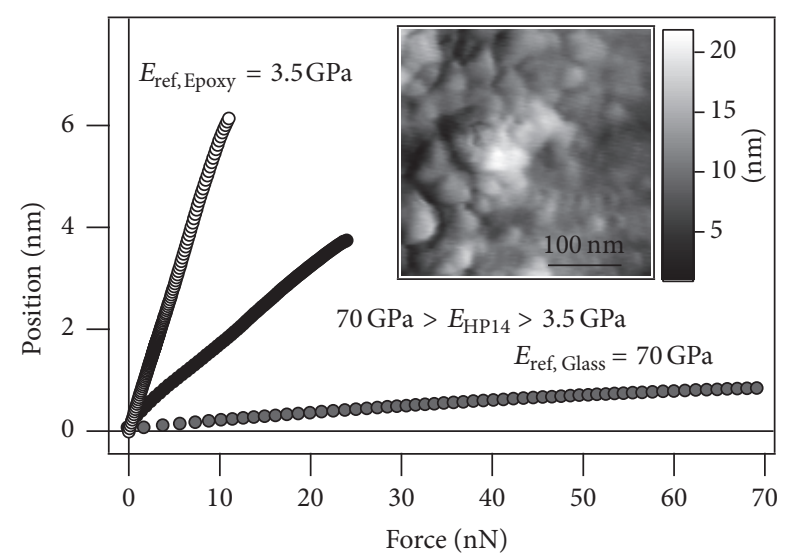

FIGURE 4: Average ADFS curves corresponding to HP14 (filled circles), epoxy matrix (blank circles), and glass (grey circles) with Hertz fit corresponding to $70 \mathrm{GPa}$ (black line). Inset topography of a HP14 nanoparticle (elevated structure left to the center) embedded in an epoxy matrix. At each pixel, an ADFS curve was recorded.

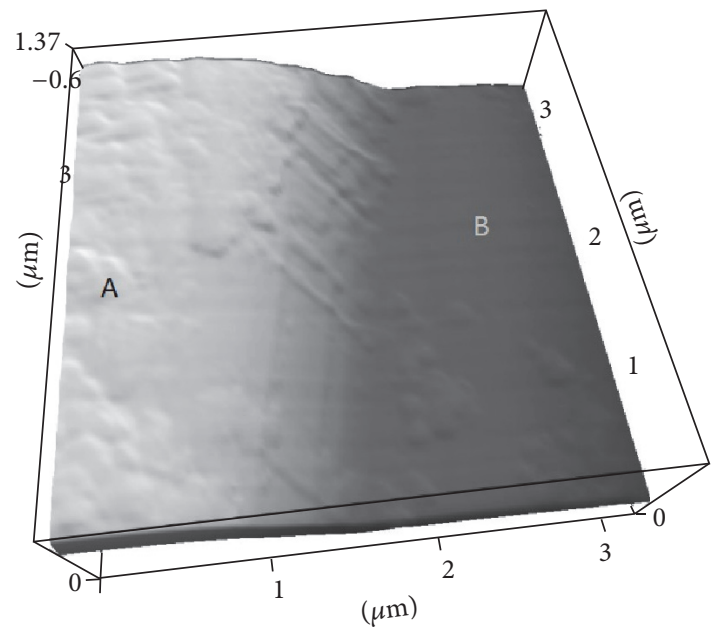

Figure 5: Tapping mode topography of GeoB.

The measurement of embedded HP14 particles was repeated successfully for at least 10 times and the stiffness of the particle never exceeded the stiffness of glass.

In order to confirm this unexpected compliance of boehmite, a macroscopic crystal GeoB was measured by FDC. The AFM probe used for this measurement was a Nanosensors Pointprobe-Plus-NCHR (Nanosensors, Neuchatel, Switzerland) (cf. Table 1). For reference, FDC and ADFS measurements on glass substrates and epoxy were conducted.

FDC measurements of GeoB were conducted on a plateau step, which means that forces were applied roughly perpendicular to the octahedral double planes. This corresponds to the simulated FDC of boehmite shown in Section 4. As confirmed by Raman spectroscopy, the GeoB crystal shows little to no defects. However, as can be seen in the topography of GeoB, shown in Figure 5, the surface of the sample is contaminated with amorphous material. It cannot be determined if this amorphous material is boehmite. Only curves

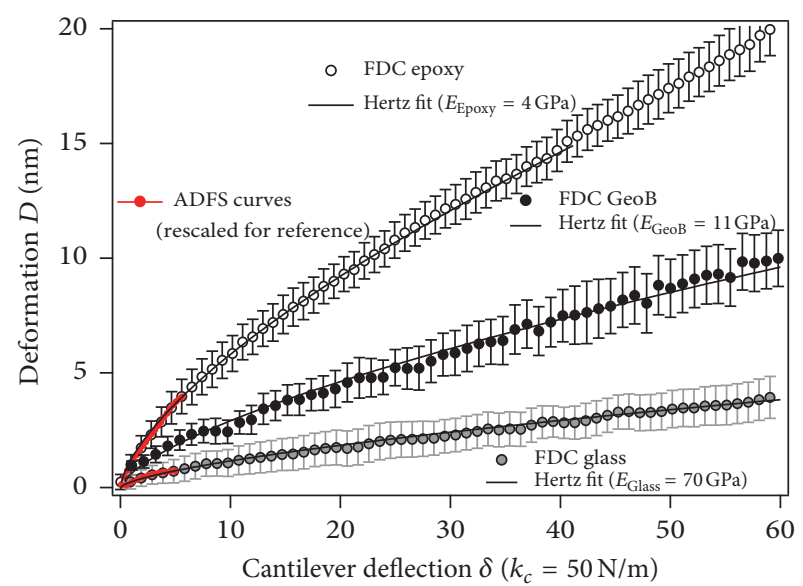

FIGURE 6: Deformation versus cantilever deflection: experimental curves of glass (grey circles), GeoB (filled circles), and epoxy (blank circles). Standard deviation of the average curve is shown as error bars. The Hertz fit of each experimental curve yields Young's modulus. For comparison, the ADFS curves on glass and epoxy were rescaled and added to the plot (red markers).

on clean spots of the sample were accepted and averaged. From averaged FDCs, the deformation was calculated from the cantilever deflection and the sample position and plotted versus the cantilever deflection which is proportional to the applied force (GeoB curve in Figure 6). These experimental curves can be fitted by Hertz, (1), yielding a reduced Young's modulus $E_{\text {tot }}$. Applying (2), Young's moduli $E_{\text {Epoxy }}$ and $E_{\mathrm{GeoB}}$ can be calculated, assuming Poisson's ratios for epoxy and boehmite to be $v_{\text {Epoxy }}=0.3$ and $v_{\mathrm{GeoB}}=0.25$. The AFM tip's elastic constants are $E_{\text {tip }}=245 \mathrm{GPa}$ and $v_{\text {tip }}=0.25$.

The hysteresis of the approaching and the retracting part of the FDC was considered to reveal inelastic deformations.

FDCs at two different spots (A and B in Figure 5) on $\mathrm{GeoB}$ are shown in Figure 7. It should be noted that the deformations at an uncontaminated spot (B) are solely elastic, since there is no hysteresis apparent and only such hysteresis free curves were accepted for generating the average curve shown in Figure 6. It should be also noted that all curves shown in Figure 6 are in agreement with the Hertz theory, which also implies that only elastic deformations are induced, since the Hertz theory only considers elastic deformations.

The curves shown in Figure 6 are also in very good agreement, considering shape and relation to each other, with the ADFS curves of the experiment concerning the HP14 shown in Figure 4.

Furthermore, boehmite with a very low degree of crystallinity (hyB) was examined in order to determine if the unexpected compliance of boehmite is due to the presence of lattice defects and amorphous domains. The AFM probe used for this measurement was a Mikromasch HQ:NSC35 (Wetzlar, Germany) (cf. Table 1). For reference, FDC measurements on glass substrates were conducted.

The topography of hyB (Figure 8 ) shows partly crystalline sheets on which FDC measurements can be conducted. Due to the signal to noise ratio of single FDCs, it is preferable to calculate an average curve from a number of FDCs. 


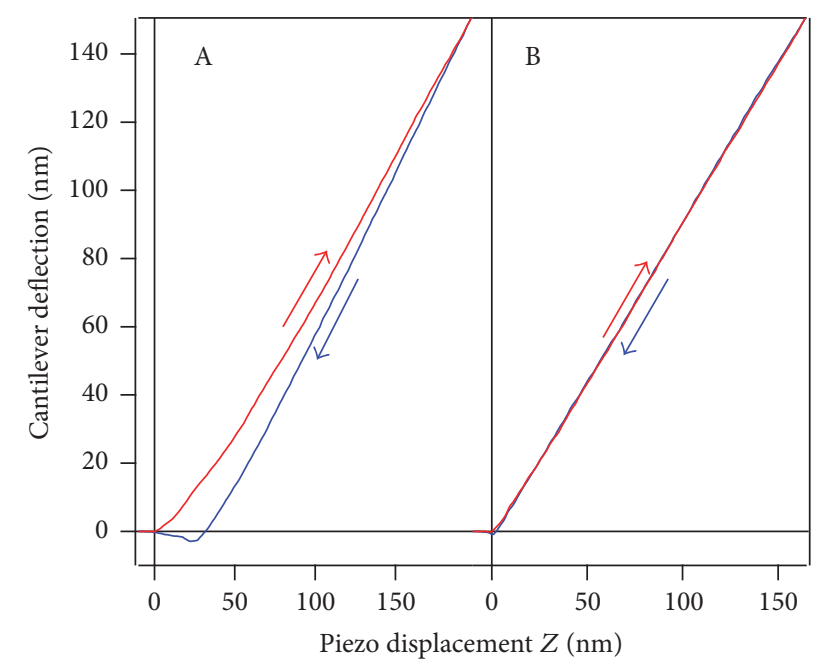

FIGURE 7: FDCs (approach red, retract blue) taken on the spots A (contaminated) and B (clean) in Figure 5. Curve A shows a considerable hysteresis. Curve B shows no hysteresis; therefore, purely elastic deformations can be assumed.

To collect enough curves, which were all recorded under comparable conditions, curves were measured successively at one point and an average was calculated from approximately 10 loading/unloading cycles. From these average curves, the deformation was calculated from the cantilever deflection and the sample position. The deformations from three different spots (A, B, and C) are shown in Figure 9 versus the cantilever deflection. Since curves taken on different spots vary a lot and we solely want to demonstrate a characteristic behavior of curves, we do not show the standard deviation. Also shown are the resulting curves of a measurement of glass and a calculated Hertz curve for epoxy. These deformation curves cannot be fitted by the Hertz relation (1) and, therefore, indicate inelastic behavior. Especially at low applied forces (up to $10 \mathrm{~nm}$ cantilever deflection), they show deformations which even exceed the deformations measured on epoxy. At higher forces, the slope of the deformation curve decreases, which indicates stiffer properties.

This is confirmed by analyzing the approach and retraction curve, shown in Figures 9 and 10, for the spots A, B, and C. At low forces, a high hysteresis indicates large inelastic deformations. At higher forces, the material underneath the tip is already compressed and behaves more elastically. This is a characteristic behavior, when a thin compliant layer on top of a stiff substrate or mechanical gradient (stiffness increases with distance to the surface) is measured. In this case, the Hertz theory does not apply, since Young's modulus is not a constant; it depends on the deformation $D$.

3.4. Summary of Experimental Part. These experiments were conducted to decide on the cause for the low experimental Young's modulus, measured on boehmite nanoparticles: Is it a defect of the crystal or is the crystal indeed that compliant? Amorphous domains would be stable at the surface of the boehmite crystal since lattice defects are expected to decrease towards the center of a crystal and the nanoparticles have been shown to be crystalline. Thus, amorphous boehmite or pseudoboehmite would form a compliant layer on the outside of the crystal. If an amorphous phase was present in the measurement of HP14, shown in Section 3.3, it would contribute to the mechanical measurements in the same way as shown in measurements of hyB. That means that FDC or ADFS curves would show a much higher slope and hysteresis at the very beginning of the curve. On the contrary, ADFS curves taken on HP14 show very similar characteristics to the FDCs taken on GeoB, which are in agreement with the Hertz theory, by showing no gradient in Young's modulus. Therefore, slippage of the planes appears as a more likely explanation for the low measured stiffness, which is not in accordance with the measurements on GeoB, where deformations were found to be elastic. The authors hypothesize that in the case of a macroscopic, monocrystalline boehmite (GeoB) the induced deformation leads to local lattice distortions due to plane slippage which will be equilibrated by the far-field lattice structure after the applied force is removed. This is only possible because (a) the (010) layers are rather stiff in comparison with the weak bonds between the layers and (b) the volume effected by the deformation is small compared to the monocrystalline domain. The compression created in the (010) layers due to the deformation is spread along the whole plane and, acting like a spring, can restore the lattice structure in the deformed volume. The deformations, therefore, appear to be elastic. In case of the nanoparticles, deformations are permanent since there is no unaffected far-field lattice structure. However, for both the micro- and macroscopic boehmite, the measured stiffness is much lower than what is expected due to plane slippage.

In fact, ambivalent behavior of boehmite nanoparticles are supported by literature. Halbach and Mülhaupt [26] found a considerable increase of the elastic modulus of boehmite-based polyethylene nanocomposites (which is a strong indication for high Young's modulus of boehmite) but also observed no decrease in elongation break, which is usually associated with brittle filler material. Also Khumalo et al. [27] investigated the rheological properties of polyethylene/boehmite nanocomposites and found an uncharacteristic lack of increase in the melt viscosity of such materials. In order to get a better understanding of these discrepancies, a numerical study of the problem was pursued.

\section{Numerical AFM Simulation}

The results presented in the following are the outcome of computational studies simulating an AFM FDC measurement on a pure boehmite crystal structure and combined boehmite-epoxy structures, respectively.

4.1. AFM Simulation Models. All simulations are carried out using the Molecular Dynamic Finite Element Method (MDFEM, for a detailed presentation see $[28,29]$ ). The MDFEM provides a framework for calculating classical molecular dynamics (MD) problems within the wellestablished Finite Element Method (FEM) (see, e.g., Wackerfuss [29] for an overview of molecular dynamics in the context of the FEM). The main motivation for this approach 


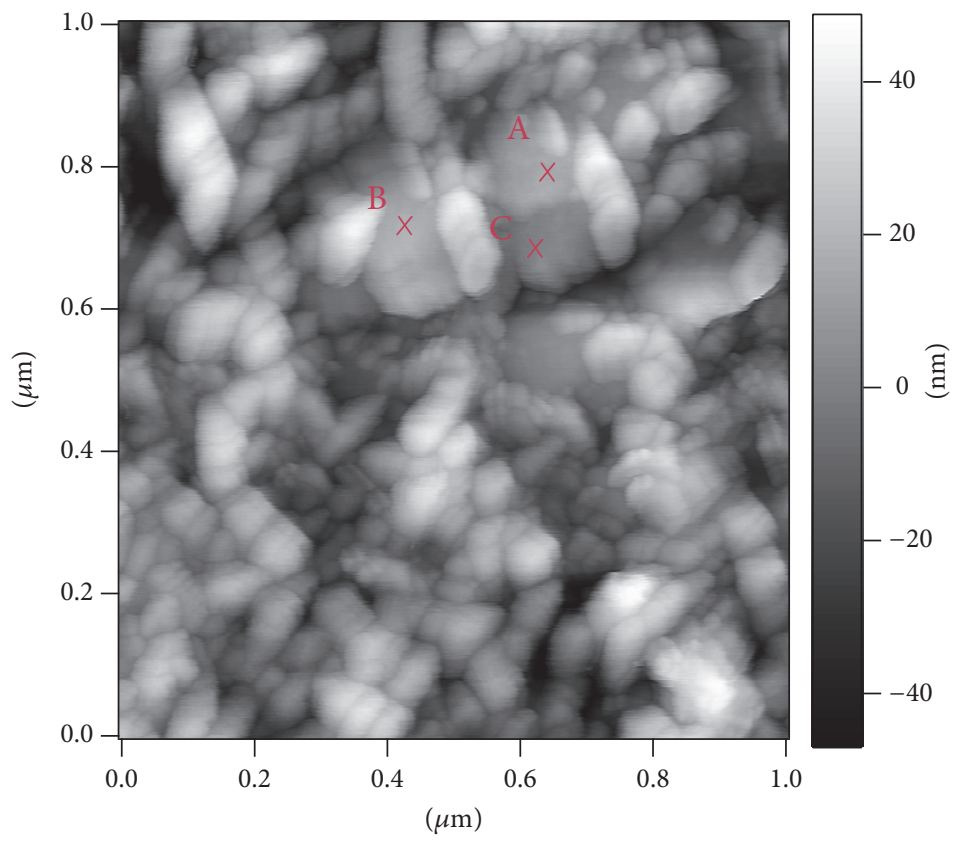

FIgURE 8: Tapping mode topography of hyB.

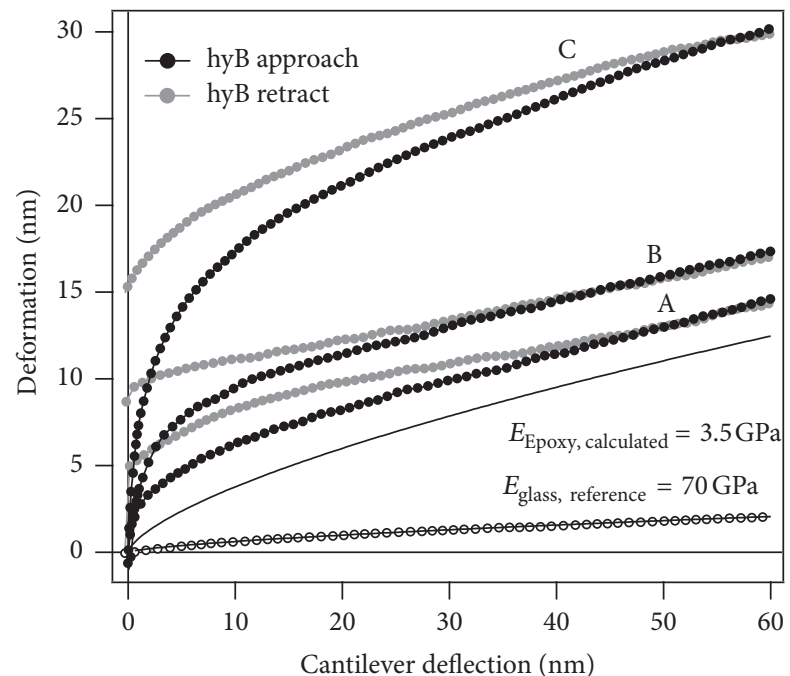

FIGURE 9: Deformation-deflection curves, derived from averaged FDC, measured on glass (blank circles) with its Hertz fit (black line) and hyB (approach black circles, retract grey circles) at the spots A, $B$, and $C$. The Hertz curve of epoxy was calculated and is shown for comparison (black line).

is the simplification of multiscale techniques, as in particular the coupling of atomistic and continuum mechanics simulations can be performed in a very efficient way within one software package. Additionally, the need for special in-house developed software for the MDFEM interaction is reduced, since the highly efficient solvers as well as the pre- and postprocessing tools of the FEM software can be used.

The MDFEM requires special FE-meshes that are generated with an in-house software package (using Open Babel

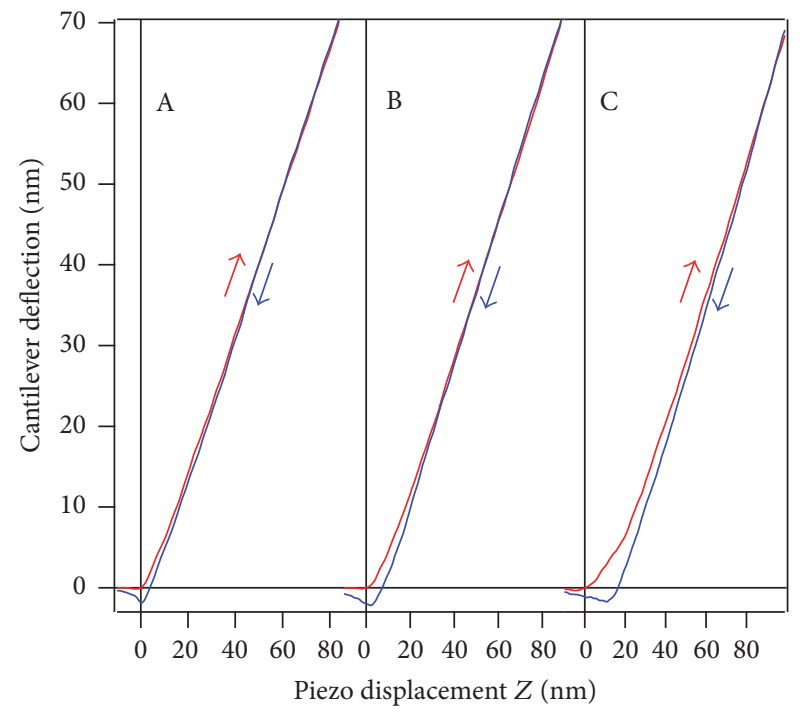

Figure 10: FDC curves (approach red, retract blue) taken on the spots A, B, and C in Figure 11.

[30] and Packmol [31]) based on the chemical structure of the constituents. The boehmite is assumed to be of $\mathrm{cmcm}$ unit cell $(a=2.8681 \AA, b=12.2256 \AA$, and $c=3.6941 \AA)$, as reported by Bokhimi et al. [8] and experimentally confirmed through X-ray diffraction as discussed in Section 3.1. The AFM tip material is silicon (Fd3m unit cell, $a=3.5668 \AA$ ). Starting from unit cells, the model is generated by duplicating the unit cells and extracting the desired geometry. In case of the matrix material, Bisphenol-A-diglycidylether (DGEBPA) prepolymer chains are packed randomly into the simulation 

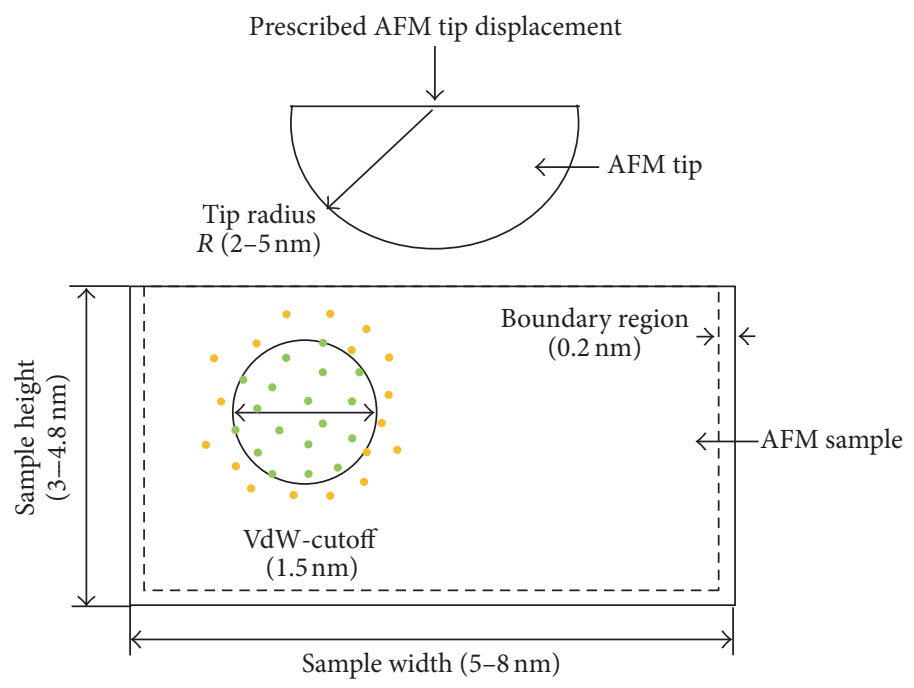

FIGURE 11: Schematic representation of the AFM models.

box avoiding intersections with the particle. Subsequently, the matrix is cured by randomly iterating over all hydroxyl groups, searching for the closest epoxy group and connecting both reactive sites using the Methyltetrahydrophthalic Anhydride (MTHPA) hardener molecules. If all hardener molecules have been placed or no more reaction partners are found, the same process is executed for the etherification reaction, connecting reactive sites with a slightly bigger distance without a hardener molecule. Figure 11 shows a schematic representation of the AFM models.

All simulations presented in the following are performed using the DREIDING force field [32]. It has been parameterized for a large subset of the periodic tables of the chemical elements and was recently used successfully to model anhydride-cured epoxy resin as well as alumina [33] and boehmite nanoparticles [34] in an MDFEM context. Even though it is possible to apply different cutoff radii for the physical interactions (denoted as VdW-cutoff) within the sample and between sample and tip, a spherical cutoff of $15 \AA$ has proven to be suitable for both cases by parametric studies. The AFM tip is modeled as an ideal hemisphere and is considered to be rigid, meaning that all chemical and physical interactions are eliminated within the tip by removing the corresponding elements and introducing a rigid coupling to a reference node. Preliminary investigations have shown that this simplification has negligible influence on the resulting elastic modulus. The first step in the simulations is, as usual in molecular dynamics calculations, the determination of the equilibrium state of the system. Therefore, the whole model including the AFM tip is relaxed over a period of time ranging from 50 to $100 \mathrm{ps}$, depending on the model size. The time step for all calculations is determined dynamically using the bond stiffness and lies in the range of 1-2 fs. In the relaxation step, the equilibrium position of the tip (in the sense of the tip lying on the sample surface without cantilever forces) is obtained automatically. Subsequently, the load is applied quasistatically in terms of an AFM tip displacement of 0.5 to
$1.5 \mathrm{~nm}$, depending on the sample and tip size. A simulation time of 300 ps for the maximum displacement of $1.5 \mathrm{~nm}$ has proven to be suitable by parametric studies. All samples are constrained on all surfaces in a border region of $0.2 \mathrm{~nm}$, except for the top surface, where the tip indents the material.

Due to the comparatively high numerical effort of $\mathrm{MD}$ simulations as well as MDFEM simulations, it is necessary to reduce the model size. Thus, in preliminary investigations, the effect of the tip radius and the sample size on the calculated modulus is analyzed.

4.2. Results of Preliminary Simulations. The indentation direction is parallel to [010] in all simulations of this subsection. As a result of the simulations, force-deformation curves are obtained. Young's modulus is then calculated via a Hertz fit in a manner similar to the AFM experiments. If a rigid AFM tip is assumed, $E_{\text {tip }}$ becomes infinite and the first term in the parenthesis in (2) vanishes. The reduced Hertz curve is then fitted to the simulated curve with a least square fit using MATLAB.

Firstly, the influence of the tip radius on the calculated elastic modulus of the perfect boehmite structure is examined. Therefore, the tip radius is increased from 1 to $5 \mathrm{~nm}$ in steps of $1 \mathrm{~nm}$. The sample size is chosen to be four times as big as the tip radius. This is shown to be large enough not to impose any boundary effects on the measured modulus later on. The results of this study are displayed in Figure 12. It is apparent that with increasing tip radius the calculated moduli converge. Small tip radii underestimate the moduli, but yet for the $2 \mathrm{~nm}$ tip, the deviations from the results of bigger tips are smaller than $4 \%$. Therefore, the $2 \mathrm{~nm}$ tip has been chosen for further investigations. Furthermore, it is apparent that the calculated values (127-135 GPa) lie slightly above the shown reference value from experimental tests from the literature (120 GPa [12] difference approx. 13\%). However, the applicability of this source in the present context is discussed in Section 1. 


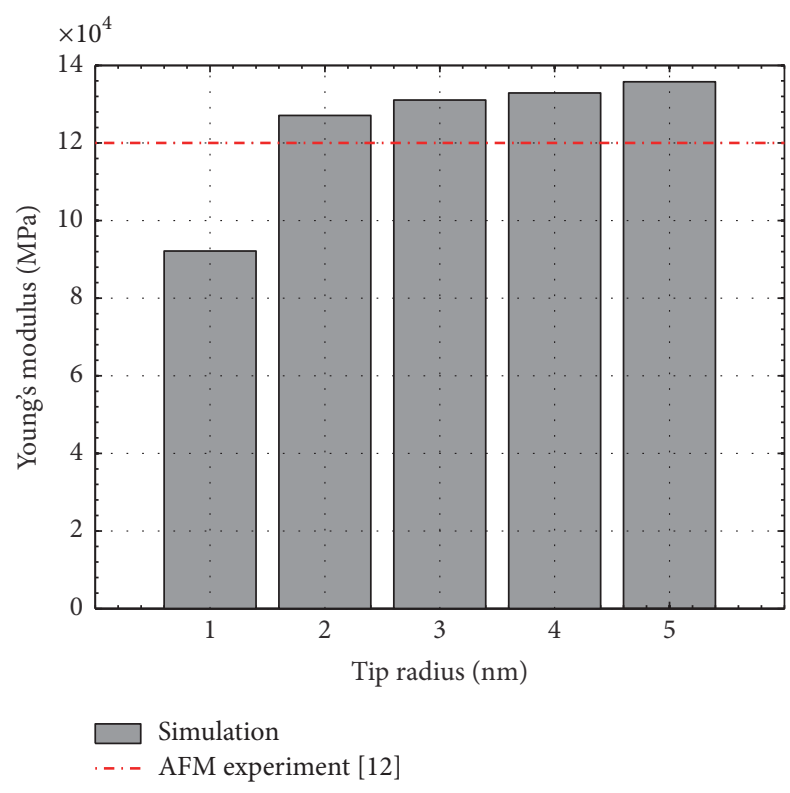

FIGURE 12: Influence of the AFM tip size on the calculated elastic modulus of the perfect boehmite crystal.

Secondly, the sample size is addressed with a similar approach. Starting from $8 \times 4.8 \times 8 \mathrm{~nm}$, the sample size is gradually reduced to a minimum of $5 \times 3 \times 5 \mathrm{~nm}$. The tip size is fixed with $2 \mathrm{~nm}$. The resulting force-deformation curves are shown in Figure 13. The straight lines in Figure 13 indicate the onset of a deviation between the fitted curve and the simulated curves. This effect is caused by boundary effects (at a certain penetration, the rigid boundaries start influencing the curve), indicating that the sample size is chosen too small. To avoid this, either the sample size has to be increased or the range in which the Hertz fit is calculated has to be truncated (here, the latter was already performed).

As expected, Figure 13 shows that the point where the simulated curves start deviating from the Hertz curve is shifted to a lower deformation with decreasing sample size. Interestingly, reducing the model size from 8 to $6.5 \mathrm{~nm}$ and from 6.5 to $5 \mathrm{~nm}$ leads to an almost equal deformation shift of about $0.15 \mathrm{~nm}$. The sample size of $8 \times 4.8 \times 8 \mathrm{~nm}$ is chosen as the minimum required sample size in order to receive a sufficiently large interval for the Hertz fit.

4.3. Simulation Results. As can be concluded from the previous section, the elastic modulus of boehmite is converging to a value of approximately $136 \mathrm{GPa}$ and calculated with a value of $127 \mathrm{GPa}$ for a tip size of $2 \mathrm{~nm}$, which is the reference model for further investigations.

Until here, all simulations have been performed in the [010] direction perpendicular to the boehmite layers. In the simulation coordinate system, this corresponds to the $b$-direction (see Figure 1). To account for the anisotropy, additional simulations were performed in [100] direction ( $a$ direction) and [001] direction (c-direction), resulting in a Young's modulus of $232 \mathrm{GPa}$ and $267 \mathrm{GPa}$, respectively.

The moduli obtained from the simulations strongly differ from the experimentally obtained values (in the range of

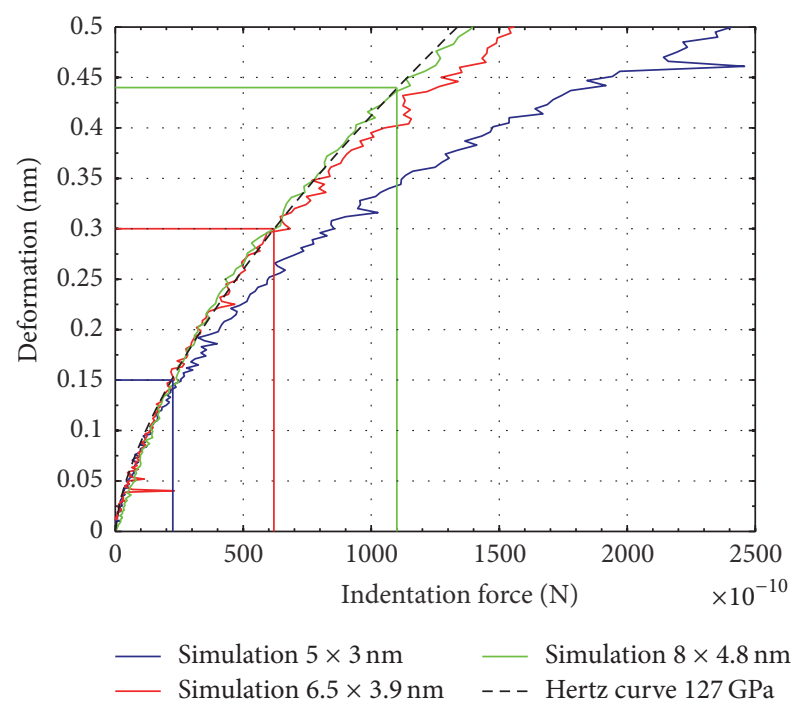

FIGURE 13: Effect of the sample size on the calculated elastic modulus of the perfect boehmite crystal. Only data left of the vertical line in the respective color was considered in the fit.

$10 \mathrm{GPa}$ for GeoB). To identify the cause of these differences, in the following, the influence of several imperfections is investigated. Since the indentation in [010] direction resulted in the softest behavior so far, the following investigations are performed perpendicular to the crystal layers only. Firstly, the intercalation of water between the boehmite layers that was reported by Bokhimi et al. [8] is addressed. Therefore, $10 \%$ of the alumina atoms of the interlayer surface of the boehmite sample are modeled being occupied with a water molecule bonded by a hydrogen bond instead of just a hydroxyl group. This leads to slightly increased interlayer distances and thus to a weaker bonding between the layers. However, the reduction obtained is in the range of $16 \%$, resulting in an elastic modulus of approximately $113 \mathrm{GPa}$.

Furthermore, the influence of alumina and hydroxyl group defects is examined. To consider defects, random Alatoms or $\mathrm{OH}$-groups are deleted from the model, assuming that the defects do not lead to fundamental changes in the crystal structure. In both cases, the amount of defects is varied from 0 to $20 \%$ in steps of $5 \%$. For each variation, the outcome of three calculations is averaged. The resulting values are shown in Figure 14. It is apparent that hydroxyl group defects lead to rather small reduction of the modulus by a maximum of $18 \%$ for $20 \%$ deleted $\mathrm{OH}$-groups. The cause of the softer behavior is the decrease of hydrogen bonds existing in the model that in turn lead to a weaker bonding between the boehmite layers. In case of alumina defects, the reduction is much more distinct. For the case of $20 \%$ deleted $\mathrm{Al}$-atoms, the reduction of the elastic modulus is in the range of $47 \%$, resulting in a value of about $72 \mathrm{GPa}$. This comparatively large reduction is caused by voids in the octahedral boehmite crystal structure. However, both imperfect models still result in much higher moduli than the experimentally determined ones. However, the net-like structure of the hydrothermally formed boehmite film, which 


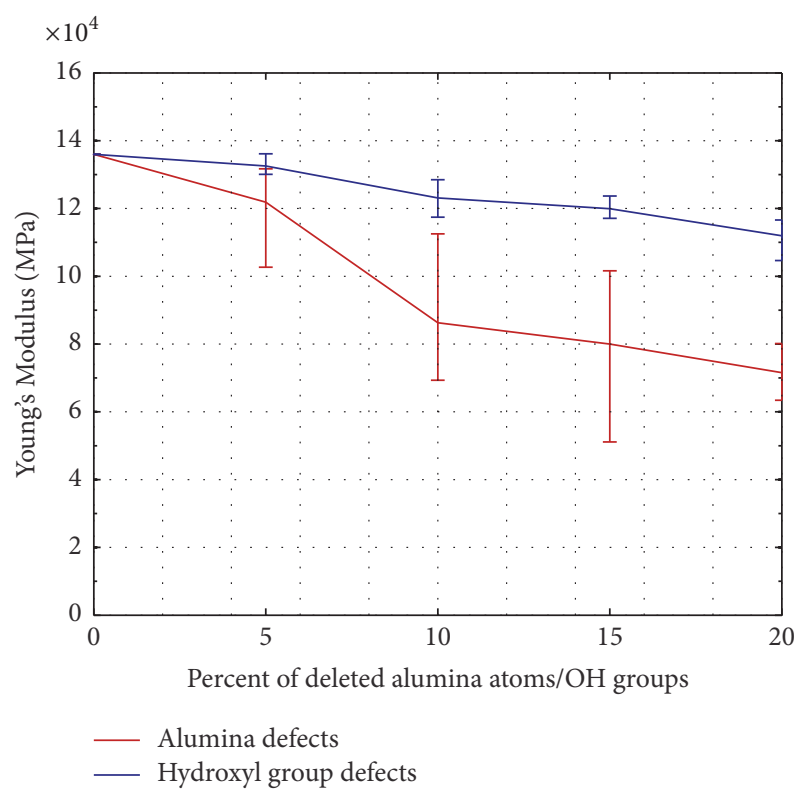

FIGURE 14: Influence of alumina/hydroxyl group defects on the calculated elastic modulus of boehmite.

was observed in the experiments, can induce additional mechanisms that were not covered by the simulation. This includes, for instance, cracking of layers or the tilting of bigger structures like needles or plates. The explicated phenomena can further reduce the resulting modulus.

The drastically reduced moduli obtained from experimental AFM measurements have been interpreted to originate from inelastic phenomena. These include, in particular, slippage of the crystal layers but also cracking of layers and tilting of substructures. To provide evidence for this inelastic behavior, an attempt is made by performing AFM simulations on combined boehmite/epoxy samples. The crystal is modeled with the real orthorhombic shape, different particle orientations, and a particle size of $5 \mathrm{~nm}$, as illustrated in Figures 15(a)-15(c). This again requires a reduction of the model size (the real primary particle size is in the range of $14 \mathrm{~nm}$ [18]) in order to limit the numerical effort. The box size perpendicular to the loading direction is chosen based on findings from preliminary investigations on the pure matrix material as $15 \mathrm{~nm}$. The matrix is modeled in order to keep the particle in its place and to allow for the simulation of inelastic phenomena of the boehmite particle.

The size in loading direction is assigned according to the orientation of the particle in such a way, that the boundary region is located directly below the particle (see also Figure 15(a)). This corresponds to a rigid surface supporting the particle, as an idealization of the sample holder in the real experiment. It should be mentioned that, for stability and performance reasons, the chemical bonds are modeled using harmonic potentials, which cannot reproduce the failure of bonds and hence the cracking of boehmite layers. Three different configurations are simulated: Firstly, the load is applied in [010] direction (perpendicular to the layers), secondly, the layers are oriented diagonally and the particle

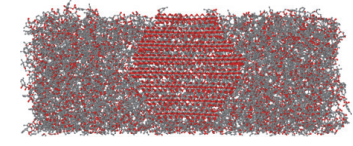

(a) Parallel to layers, plane to plane support

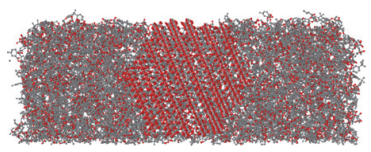

(b) Inclined layers, plane to plane support

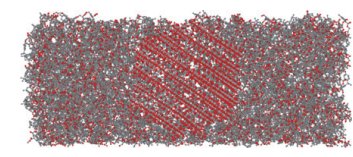

(c) Inclined layers, edge to plane support

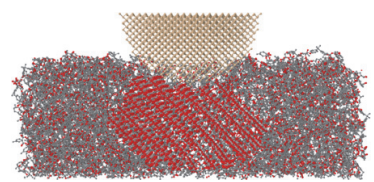

(d) Deformed configuration of (b) at peak load

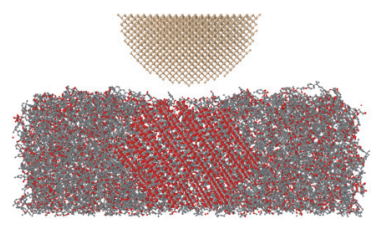

(e) Deformed configuration of (b) after unloading

FIGURE 15: (a)-(c) Investigated undeformed particle orientations. (d) and (e) Deformed configurations corresponding to the initially inclined layers with plane to plane support.

has a plane area of support (Figure 15(b)), and thirdly, the layers are oriented diagonally and the particle rests on the sample holder only by an edge; see Figure 15(c).

The three configurations have been exposed to a loading and subsequently an unloading process. Figure 16 shows the corresponding deflection versus force curves of the loading and unloading process as well as the respective Hertz fit.

A huge difference between the configuration perpendicular to the layers and the other two configurations is apparent. For loading in [010] direction, the loading and unloading path are nearly identical, indicating a purely elastic problem and resulting in a Young's modulus of approximately $136 \mathrm{GPa}$. This result shows a good agreement with the simulations performed on the pure boehmite sample. For the other particle orientations, a drastically reduced slope of the curves and a hysteretic behavior is observed. This indicates inelastic behavior. The corresponding plots of the deformed structure of the configuration with plane to plane support, Figures 15(d) and 15(e), provide insight in the inelastic mechanisms. 
The main mechanism leading to the reduced slope of the resulting curves is a slippage of the crystal layers that can clearly be seen in the deformed shape in Figure 15(d). The sudden onset of crystal layer slippage can as well be seen from the force jumps in the loading path in the deflection-force curves in Figure 16. The roughness of the curve originates from the jump from one equilibrium position of the interlayer hydrogen bonds to another. The crystal layer slippage additionally induces a tilting of the single boehmite layers. In case of the configuration with edge on plane support (see Figure 15(c)), the particle can furthermore tilt as a whole, resulting in an even more decreased slope of the curves.

In both cases, the Hertz curve is fitted to the unloading curve, since this curve is in the presence of inelastic effects expected to exhibit the elastic behavior. This leads to calculated moduli of approximately $40 \mathrm{GPa}$ for plane to plane support and $32.5 \mathrm{GPa}$ for edge to plane support. However, the unloading path is not considered to be purely elastic. The deformed structure after the unloading process in Figure 15(e) shows that the layer slippage is reversed by the unloading. This reversal is assumed to be introduced by restoring forces of the compressed matrix material as well as through the tip-particle adhesion. The unloading curve thus also exhibits small jumps. The calculated moduli from the unloading curve show a significant reduction compared to the simulation of the pure boehmite samples; that is, a significant approach to the experimental dimensions (around values of $10 \mathrm{GPa}$ ) is observed.

\section{Results and Discussion}

Results from literature suggested that the Young's modulus of boehmite falls in the range from 120 to 160 GPa. MDFEM simulations on bulk boehmite presented in Section 4.3 yielded anisotropic Young's moduli of $232 \mathrm{GPa}, 136 \mathrm{GPa}$, and $267 \mathrm{GPa}$ in the directions [100], [010], and [001], respectively. While these results are at least of similar magnitude to the results from the cited literature, experimental investigations indicated far lower stiffness. For the macroscopic GeoB sample, the stiffness obtained from measurements was found to be on the order of $10 \mathrm{GPa}$ for a test roughly aligned with the [010] direction (cf. Section 3.4). For the particle sample, the stiffness was determined to fall somewhere in between epoxy (3.5 GPa) and glass $(70 \mathrm{GPa})$ in Section 3.3. Three hypotheses for this conspicuous discrepancy were put forth and discussed: sample size related effects, inelastic slippage of crystal layers, and possible distortion of the experimental results by the presence of amorphous boehmite.

The third hypothesis was refuted in Section 3.3 by experiments on boehmite samples known to feature low crystallinity which resulted in even lower stiffness. This led to the conclusion that the experiments on particle and GeoB samples are not critically affected by the presence of amorphous boehmite. This interpretation is consistent with results from simulations on bulk boehmite including defects in Section 4.3. A number of particle imperfections were considered, such as alumina or hydroxyl defects or intercalated water molecules. In particular, the latter one is

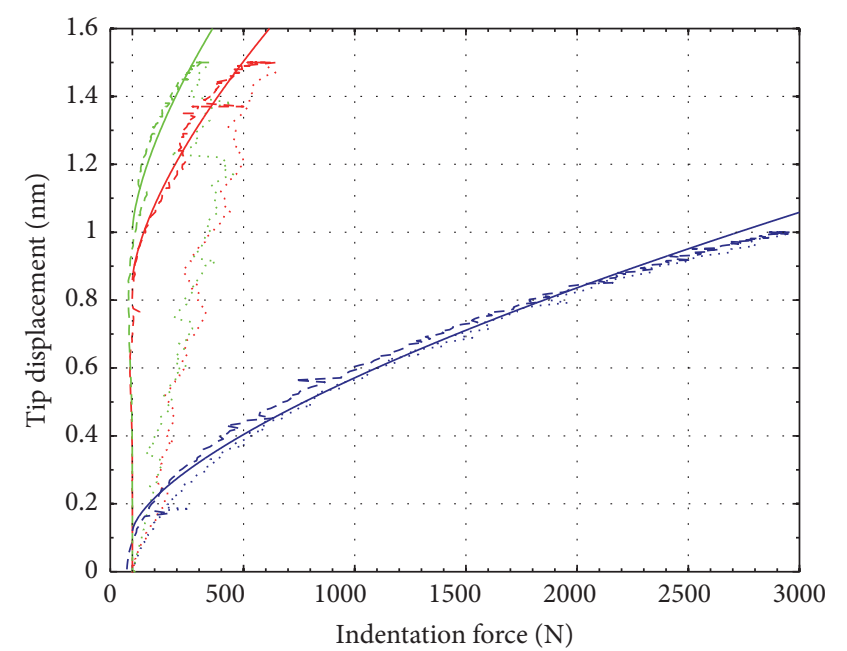

$\ldots .$. Loading perpendicular to layers
$\ldots \ldots$ Loading inclined layers, plane to plane
$\ldots \ldots$ Loading inclined layers, plane to edge
- - - Unloading perpendicular to layers
- - - Unloading inclined layers, plane to plane
- - - Unloading inclined layers, plane to edge
- Hertz fit $136 \mathrm{GPa}$
- Hertz fit $40 \mathrm{GPa}$
Hertz fit $32.5 \mathrm{GPa}$

FIGURE 16: Loading curve, unloading curve, and Hertz fit of the investigated configurations from Figure 15.

observed in the real crystalline material. However, none of the considered defects could account for a sufficient drop in stiffness.

The second hypothesis, on the other hand, is corroborated by simulations emulating the actual AFM test conditions of a particle embedded in epoxy resin. The simulated measurements resulted in substantially lower stiffness, 32.5 to $40 \mathrm{GPa}$, comparable to the experiments on GeoB, when the loading direction did not coincide with [010]. It seems likely that to some extent the stiffness mismatch can be attributed to inevitable load-misalignments in the experiments. Inelastic layer slippage triggered by misalignment of the loading direction is, therefore, identified as the probable cause of the very low stiffness in certain situations. Furthermore, the effect of imperfect contact of the particle on the sample holder was considered, also resulting in somewhat reduced apparent stiffness.

In the simulations on the particle, inelasticity is also clearly indicated by the presence of hysteresis in the loading/unloading curves. No hysteresis is apparent in the FDCs of the GeoB, though. It is presumed that in macroscopic crystal samples the slippage is reversed by conformation of the deformed areas to the far-field lattice structure after unloading. In microscopic samples, however, the entire crystal is affected by the deformation and it is, therefore, permanent. Hence, the hypothesis of a sample size dependent effect is accepted as well.

Moreover, the model size of the simulation might have an effect on the results too. Since the number of atoms had 
to be reduced for the simulation, the simulation box represents a downscaled model of the actual AFM measurement. Although the box size was selected from a series leading to convergent results, it cannot be ruled out that for a significant enlargement of the box additional phenomena like layer buckling become relevant. Further inaccuracies of the simulation model arise from the choice of the interatomic force field or the rigid representation of the AFM tip. These effects might contribute to the remaining discrepancies, that is, stiffness as low as $32.5 \mathrm{GPa}$ in the simulations versus stiffness as low as $10 \mathrm{GPa}$ in the measurements. The remaining uncertainties motivate further investigations and will be addressed in future work.

\section{Conclusion}

The anisotropic Young moduli of the single crystalline boehmite were determined by means of MDFEM simulations, yielding values of 232, 136, and $267 \mathrm{GPa}$ in the directions [100], [010], and [001], respectively. Results of AFM experiments on boehmite nanoparticles, in contrast, showed drastically reduced but seemingly elastic moduli in the range of 10 GPa. Three hypothesized causes of the significantly lower values were examined by experiments on complementary types of boehmite, that is, geological and hydrothermally synthesized samples and further simulations of imperfect crystals and combined boehmite/epoxy models. It is concluded from these investigations that the mechanical behavior of boehmite nanoparticles and geological boehmite is dominated by the slippage of weakly linked boehmite layers. The approaching and retracting part of the experimentally determined FDC of the geological sample exhibit no visible hysteresis and hence the behavior appears to be purely elastic. However, further simulations indicated that the inelastic process of layer slippage can be reversed by certain effects. For the geological sample, in which the deformations were small compared to the size of the crystal, we hypothesize the conformation of the deformed areas to the far-field lattice structure.

\section{Competing Interests}

The authors declare that they have no competing interests.

\section{Acknowledgments}

This work originates from the Research Unit FOR 2021 "Acting Principles of Nano-Scaled Matrix Additives for Composite Structures" funded by the German Research Foundation (DFG). The authors would like to thank Dr. Franziska Emmerling, BAM, Berlin, Division 1.3, for providing and analyzing X-ray diffraction data of boehmite nanoparticles. Furthermore, the authors acknowledge the support by the RRZN scientific computing cluster, which is funded by the Leibniz Universität Hannover, the Lower Saxony Ministry of Science and Culture (MWK), and the German Research Foundation (DFG). The authors wish to express their gratitude for the financial support.

\section{References}

[1] C.-H. Chen, J.-Y. Jian, and F.-S. Yen, "Preparation and characterization of epoxy $/ \gamma$-aluminum oxide nanocomposites," Composites Part A: Applied Science and Manufacturing, vol. 40, no. 4, pp. 463-468, 2009.

[2] R. A. Horch, N. Shahid, A. S. Mistry, M. D. Timmer, A. G. Mikos, and A. R. Barron, "Nanoreinforcement of poly(propylene fumarate)-based networks with surface modified alumoxane nanoparticles for bone tissue engineering," Biomacromolecules, vol. 5, no. 5, pp. 1990-1998, 2004.

[3] S. Pradhan, R. Lach, H. H. Le, W. Grellmann, H. Radusch, and R. Adhikari, "Effect of filler dimensionality on mechanical properties of nanofiller reinforced polyolefin elastomers," ISRN Polymer Science, vol. 2013, Article ID 284504, 9 pages, 2013.

[4] C. Arlt, Wirkungsweisen nanoskaliger Böhmite in einem Polymer und seinem Kohlenstoggfaserverbund unter Druckbelastung [dissertation], 2011.

[5] W. W. Chen, S. W. Wu, Y. D. Lei et al., "Interfacial structure and performance of rubber/boehmite nanocomposites modified by methacrylic acid," Polymer, vol. 52, no. 19, pp. 4387-4395, 2011.

[6] C. Özdilek, K. Kazimierczak, D. van der Beek, and S. J. Picken, "Preparation and properties of polyamide-6-boehmite nanocomposites," Polymer, vol. 45, no. 15, pp. 5207-5214, 2004.

[7] N. Shahid, R. G. Villate, and A. R. Barron, "Chemically functionalized alumina nanoparticle effect on carbon fiber/epoxy composites," Composites Science and Technology, vol. 65, no. 14, pp. 2250-2258, 2005.

[8] X. Bokhimi, J. A. Toledo-Antonio, M. L. Guzmán-Castillo, and F. Hernández-Beltrán, "Relationship between crystallite size and bond lengths in boehmite," Journal of Solid State Chemistry, vol. 159, no. 1, pp. 32-40, 2001.

[9] A. B. Kiss, G. Keresztury, and L. Farkas, "Raman and Irspectra and structure of boehmite ( $\gamma$-Alooh) - evidence for the recently discarded D- ${ }_{2 h}^{17}$ space group," Spectrochimica Acta Part A-Molecular and Biomolecular Spectroscopy, vol. 36, no. 7, pp. 653-658, 1980.

[10] D. Tunega, H. Pasalić, M. H. Gerzabek, and H. Lischka, "Theoretical study of structural, mechanical and spectroscopic properties of boehmite ( $\gamma$-AlOOH)," Journal of Physics: Condensed Matter, vol. 23, no. 40, Article ID 404201, 2011.

[11] Y. Noel, R. Demichelis, F. Pascale, P. Ugliengo, R. Orlando, and R. Dovesi, "Ab initio quantum mechanical study of $\gamma$ AlOOH boehmite: structure and vibrational spectrum," Physics and Chemistry of Minerals, vol. 36, no. 1, pp. 47-59, 2009.

[12] R. C. Streller, Boehmite als Nanofüllstoffe für PolypropylenNanocomposites und Nanopartikel-modifizierte Polypropylen/ Kautschuk-Blends [Dissertation], 2008.

[13] M. Digne, P. Sautet, P. Raybaud, H. Toulhoat, and E. Artacho, "Structure and stability of aluminum hydroxides: a theoretical study," Journal of Physical Chemistry B, vol. 106, no. 20, pp. 5155$5162,2002$.

[14] "Jmol: an open-source Java viewer for chemical structures in 3D," http://www.jmol.org/.

[15] J. Q. Wang, J. L. Liu, X. Y. Liu, M. H. Qiao, Y. Pei, and K. N. Fan, "Hydrothermal transformation of bayerite to boehmite," Science of Advanced Materials, vol. 1, pp. 77-85, 2009.

[16] C. J. Doss and R. Zallen, "Raman studies of sol-gel alumina: finite-size effects in nanocrystalline $\mathrm{AlO}(\mathrm{OH})$," Physical Review $B$, vol. 48, no. 21, pp. 15626-15637, 1993. 
[17] K. Okada, T. Nagashima, Y. Kameshima, A. Yasumori, and T. Tsukada, "Relationship between formation conditions, properties, and crystallite size of boehmite," Journal of Colloid and Interface Science, vol. 253, no. 2, pp. 308-314, 2002.

[18] Sasol, "Dispersal HP14," Brunsbüttel, Germany, 2014.

[19] A. Guinier, X-Ray Diffraction in Crystals, Imperfect Crystals, and Amorphous Bodies, Dover Publications, New York, NY, USA, 1994.

[20] “The Rruff project,” Bob Downs, http://rruff.info.

[21] J. Yang and R. L. Frost, "Synthesis and characterization of boehmite nanofibers," Research Letters in Inorganic Chemistry, vol. 2008, Article ID 602198, 4 pages, 2008.

[22] B. Cappella, S. K. Kaliappan, and H. Sturm, "Using AFM force-distance curves to study the glass-to-rubber transition of amorphous polymers and their elastic-plastic properties as a function of temperature," Macromolecules, vol. 38, no. 5, pp. 1874-1881, 2005.

[23] D. Silbernagl and B. Cappella, "Reconstruction of a hidden topography by single AFM force-distance curves," Surface Science, vol. 603, no. 16, pp. 2363-2369, 2009.

[24] H.-J. Butt, B. Cappella, and M. Kappl, "Force measurements with the atomic force microscope: technique, interpretation and applications," Surface Science Reports, vol. 59, no. 1-6, pp. 1-152, 2005.

[25] D. Platz, D. Forchheimer, E. A. Tholén, and D. B. Haviland, "Interaction imaging with amplitude-dependence force spectroscopy," Nature Communications, vol. 4, article 1360, 2013.

[26] T. S. Halbach and R. Mülhaupt, "Boehmite-based polyethylene nanocomposites prepared by in-situ polymerization," Polymer, vol. 49, no. 4, pp. 867-876, 2008.

[27] V. M. Khumalo, J. Karger-Kocsis, and R. Thomann, "Polyethylene/synthetic boehmite alumina nanocomposites: structure, thermal and rheological properties," Express Polymer Letters, vol. 4, no. 5, pp. 264-274, 2010.

[28] L. Nasdala, A. Kempe, and R. Rolfes, "Are finite elements appropriate for use in molecular dynamic simulations?" Composites Science and Technology, vol. 72, no. 9, pp. 989-1000, 2012.

[29] J. Wackerfuss, "Molecular mechanics in the context of the finite element method," International Journal for Numerical Methods in Engineering, vol. 77, no. 7, pp. 969-997, 2009.

[30] N. M. O’Boyle, M. Banck, C. A. James, C. Morley, T. Vandermeersch, and G. R. Hutchison, "Open Babel: an open chemical toolbox," Journal of Cheminformatics, vol. 3, no. 10, article 33, 2011.

[31] L. Martinez, R. Andrade, E. G. Birgin, and J. M. Martínez, "PACKMOL: a package for building initial configurations for molecular dynamics simulations," Journal of Computational Chemistry, vol. 30, no. 13, pp. 2157-2164, 2009.

[32] S. L. Mayo, B. D. Olafson, and W. A. Goddard III, "DREIDING: a generic force field for molecular simulations," The Journal of Physical Chemistry, vol. 94, no. 26, pp. 8897-8909, 1990.

[33] A. Kempe, L. Nasdala, and R. Rolfes, "Simulating size-dependency of the tensile modulus of alumina/epoxy nanocomposites using the molecular dynamic finite element method," in Proceedings of the International Conference on Mechanics of Nano, Micro and Macro Composite Structures, no. 182, pp. 1-2, June 2012.

[34] J. Fankhänel, A. Kempe, and R. Rolfes, "Simulating atomic force microscopy for the determination of the elastic properties of nanoparticle reinforced epoxy resin," in Proceedings of the 7th European Congress on Computational Methods in
Applied Sciences and Engineering (ECCOMAS Congress '16), Crete Island, Greece, June 2016, https://www.eccomas2016.org/ proceedings/pdf/7538.pdf. 

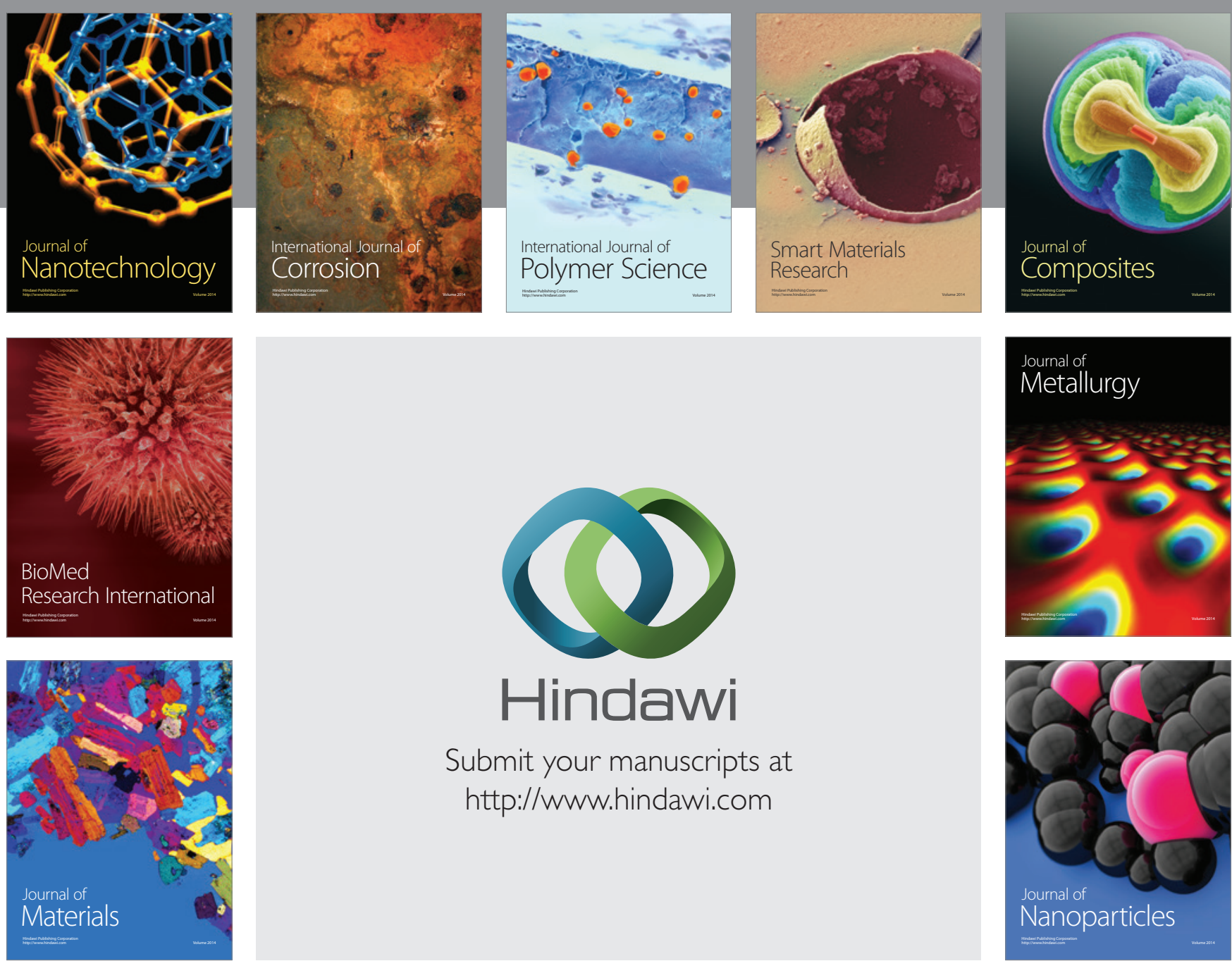

\section{Hindawi}

Submit your manuscripts at

http://www.hindawi.com

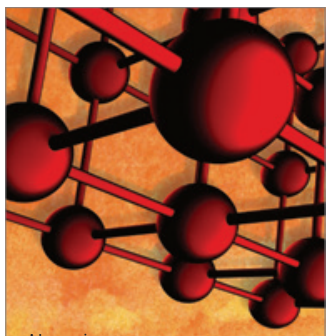

Materials Science and Engineering
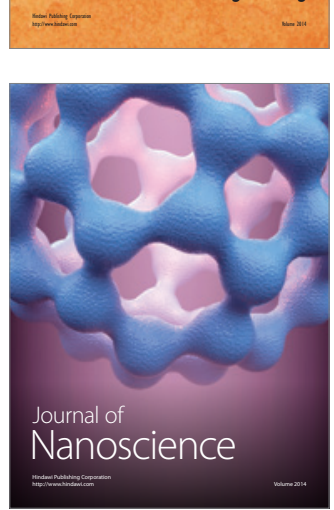
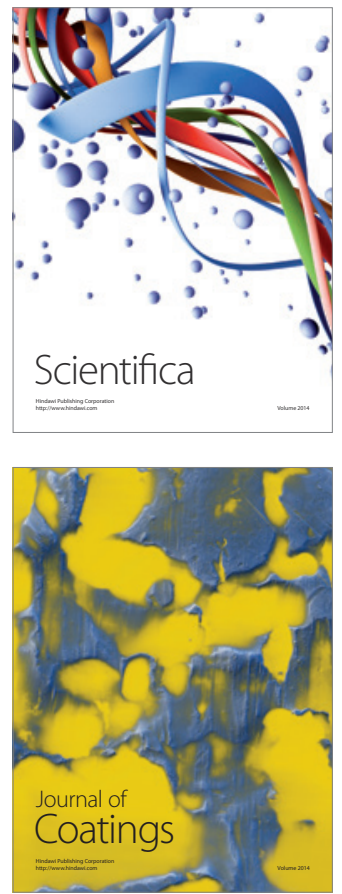
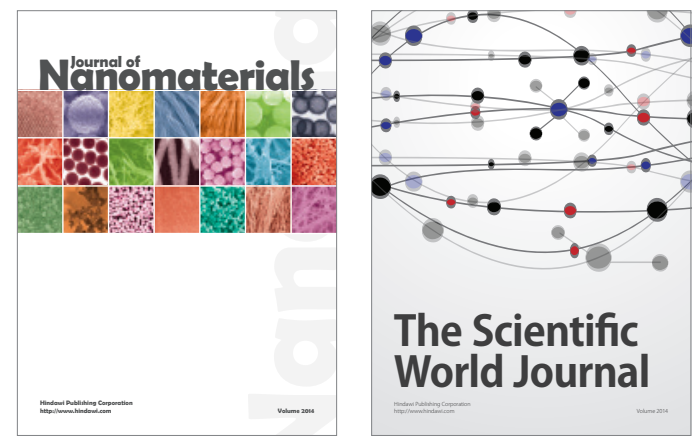

The Scientific World Journal
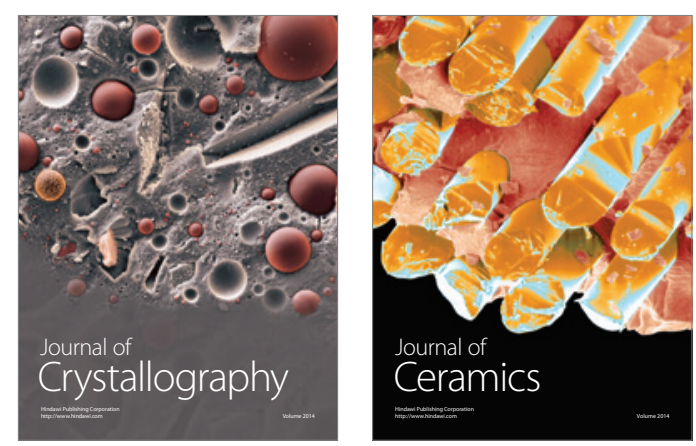
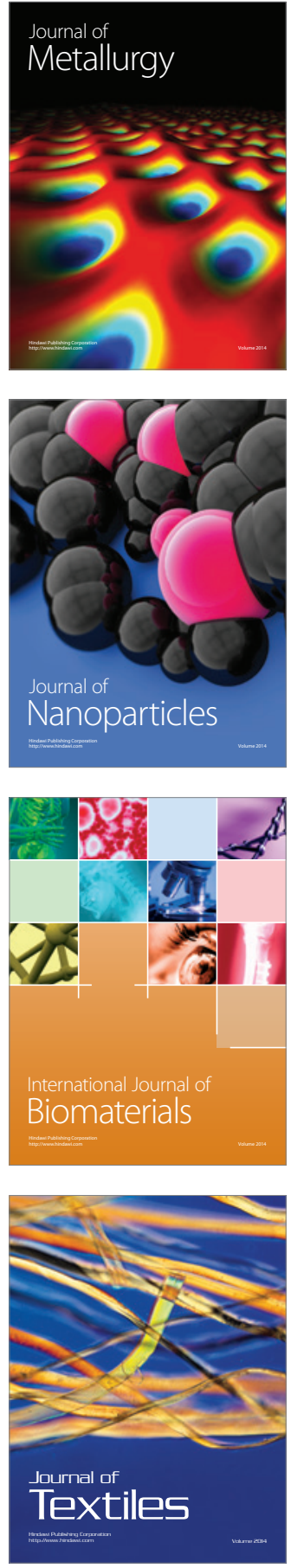